

\title{
Michelangelo's Finger: an exploration of everyday transcendence by Ray Tallis
}

\author{
Isabella Harding, 1 Katharine Harding 2 \\ 1Department of Theology and Philosophy, St \\ Brendan's VI Form College, Bristol, UK \\ 2Department of Neurology, University Hospital of \\ Wales, Cardiff, UK \\ Correspondence to Dr Katharine Harding, \\ Department of Neurology, University Hospital of \\ Wales, Cardiff CF14 4XW, UK; katharineharding@ \\ doctors.org.uk
}

We were fortunate to be joined at Book club by Ray Tallis, emeritus professor of geriatrics, poet and philosopher, to discuss his book Michelangelo's Finger Professor Tallis first conceived the book as a junior doctor in 1974 and has thought carefully about these ideas over many years of clinical practice. The book examines of the act of pointing and why it is unique to humans. Tallis uses this apparently insignificant act as the springboard for a wider discussion of what makes us human. Although the book differs from those we have previously discussed, being primarily a philosophical text, it generated a wide-ranging conversation, augmented by having the author at hand to respond to our comments.

In essence, there are two types of pointing: imperative (an instruction to get one's needs met) or declarative (to share information or an experience with another). These can be seen in young children who run about pointing and naming things so naturally. Tallis noted that children with autism use only imperative pointing, with no declarative pointing at 18 months old: a paediatric neurologist in the audience confirmed that the absence of pointing is a well known important early clinical sign of autism. We wondered how much a loss of pointing ability might contribute to communication problems for other patients, for example, those who have had a stroke, a cervical cord injury or Parkinson's disease.

We heard how philosophers have expended much time and effort considering how we can know anything about the reality of the external world when we can each know only our own perceptions. However in the everyday real world, dovetail pretty well, so Tallis's pragmatic assumption is that there is an external reality that we can conceive, aside from our merely immediate sensations. Thus we point in a space that we assume is shared by others: pointing makes no sense unless there is such a shared space. Perhaps this was another important clinical message from the book, reminding us of the importance of finding a shared viewpoint in order to facilitate communication with our patients.

We heard how language is far more than the development of primitive grunts and display and how pointing was probably closely associated with the evolution of language in humans. The initial association of primitive grunts with objects indicated directly (pointed to) would later evolve into spoken words associated with objects and ideas indicated in shared space but not necessarily visible (pointed out), and finally to language associated with ideas and emotions that could replace the gesture of pointing altogether.

We discussed the argument that pointing is not just a simple demand but rather a process of naming and informing, and how this appears unique to humans. Humans teach their young whereas other creatures rely on imitation. Additionally, language is unique in leading to new creations, and to correction, whereas other creatures are limited to their ancestral behaviour. Language has the capacity to be true or false, implying a generalised realm of possibilities; these possibilities transcend (go beyond) our sensory experience, which is limited to our position in space and time. Thus we had a picture of the animal as a prisoner of its current sensations (unable conceive of anything but the truth) and the human who can get beyond this. Michelangelo's Finger shows the fascinating overlap between philosophy and medicine, although Professor Tallis cautioned us that philosophy at this level does not help day-to-day medicine. The book provides an excellent starting point for a more substantial debate on a topic that has engaged great thinkers for centuries.

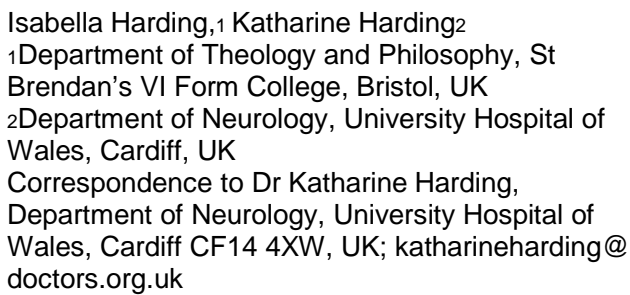

Twitter Follow Katharine Harding at @drkatharineh 
Competing interests None declared.

Provenance and peer review

Commissioned; internally peer reviewed. 